\title{
Bullous Variant of Sweet's Syndrome after Herpes Zoster Virus Infection
}

\author{
Yuichiro Endo $^{a}$ Miki Tanioka $^{a}$ Hideaki Tanizaki $^{a}$ \\ Minako Mori $^{\text {b }}$ Hiroshi Kawabata $^{b}$ Yoshiki Miyachi ${ }^{a}$ \\ Departments of ${ }^{\mathrm{a}}$ Dermatology and ${ }^{\mathrm{b}}$ Hematology, Graduate School of Medicine, \\ Kyoto University, Kyoto, Japan
}

\section{Key Words}

Bullous variant $\cdot$ Herpes virus infection · Sweet's syndrome

\begin{abstract}
Aim: Cutaneous manifestations of Sweet's syndrome (SS) are typically painful plaqueforming erythematous papules, while bullae are quite uncommon. We present a case of bullous variant of SS in acute myeloid leukaemia. In this case, herpes infection of the left mandible had preceded the development of SS.
\end{abstract}

Case Report: A 75-year-old male with myelodysplastic syndrome first presented with herpes zoster virus infection-like bullae and erosive plaques on the left side of the face and neck. Treatment with valacyclovir and antibiotics was effective only for the initial lesions, whereas the other bullae kept developing predominantly on the left side. Histopathological study revealed epidermal bulla formation, pandermal neutrophilic infiltration, erythrocyte extravasation and subepidermal oedema, but no vasculitis. The findings suggested the diagnosis of bullous variant of SS.

Discussion: Our case was unique in that bullous SS symptoms developed predominantly on one side of the cheek and neck where the herpes zoster infection occurred prior to SS. The tendency may explain the possible association between viral infection and development of SS.

\section{Introduction}

Sweet's syndrome (SS), also known as acute febrile neutrophilic dermatosis, is characterized by a rapid onset of fever, peripheral leucocytosis, and eruptions [1]. Although the precise aetiology is unknown, underlying malignancy, medication and infections have been postulated as a triggering factor of SS [1,2]. Cutaneous manifestations of SS are typically painful plaque-forming erythematous papules, while bullae are quite uncommon [2-4]. We report a case of bullous variant of SS, which 
occurred secondary to herpes virus infection of the left mandible in a patient with acute myeloid leukaemia.

\section{Case Report}

A 75-year-old male presented to our department with haemorrhagic and erosive plaques on the left side of the face and neck. Five months earlier, he was diagnosed with myelodysplastic syndrome and treated with thalidomide at the department of haematology. About 2 weeks before his referral, his myelodysplastic syndrome turned into acute myeloid leukaemia, followed by abrupt development of blisters involving the left side of the oral mucosa and cheek (fig. 1). Since herpes zoster virus infection and subsequent bacterial infection were suspected, valacyclovir and antibiotics were administered. The cutaneous blisters turned into crusts, although other erythematous plaques had newly developed on the left side of the cheek and neck and on the trunk. At the time of referral, the lesions coalesced, forming several large plaques covered with loose blisters (fig. 1, fig. 2 ). The patient's face and extremities were oedematous. Body temperature was above $38^{\circ} \mathrm{C}$ and C-reactive protein was $14.5 \mathrm{mg} / \mathrm{dl}$ (normal level: less than 0.2 ). His past medical history included treated pulmonary tuberculosis. Histopathological study of a skin biopsy specimen from the anterior cervical region revealed epidermal bulla formation, pandermal neutrophilic infiltration, erythrocyte extravasation and subepidermal oedema, but no vasculitis (fig. 3). Microorganisms were not observed by Ziehl-Nielsen, Grocott and PAS staining of a biopsy specimen. Repeated blood cultures, serum betaD glucan, polymerase chain reaction of Mycobacterium species of the skin lesion, QuantiFERON-TB2G and cytomegalovirus antigenemia $(\mathrm{C} 10, \mathrm{C} 11)$ were all negative. These findings were suggestive of SS. As systemic inflammation and renal and liver damage worsened, prednisone $40 \mathrm{mg} /$ day $(1 \mathrm{mg} / \mathrm{kg})$ was initiated. The patient's systemic and cutaneous condition improved rapidly, without scar formation after the use of steroid. The patient has agreed to the publication.

\section{Discussion}

Bullous variant is a rare subtype of SS that presents predominantly with neutrophilic bullous lesions, but not with typical erythematous plaques [2,4]. Although one retrospective study showed that vesicles and pustules can sometimes be seen to a certain degree in half of SS cases [3], bullous variant is different from usual SS in that bullae are the dominant eruptions in bullous variant. In this case, important differential diagnosis was bullous pyoderma gangrenosum [5]. However, good response to steroid therapy and healing without scar formation seem to favour the diagnosis of SS.

Cutaneous manifestations of SS are classified into the following five types: papules and nodules, plaques, bullae, ulcer and subcutaneous inflammation $[1,6]$. Caution has to be taken upon the diagnosis of bullae, ulcer and subcutaneous inflammation, since the ulcer type may mimic pyoderma gangrenosum and subcutaneous inflammation may mimic erythema nodosum. The possibility of bullous SS needs to be considered when blisters develop in febrile patients with haematological disorders.

Our case was unique in that herpes zoster virus infection seemed to trigger the development of bullous variant of SS. We think the preceding blisters were not caused by SS, but rather by the herpes zoster virus infection because the initial lesion subsided when SS progressed. Interestingly, SS developed predominantly on one side of the cheek and neck where the herpes zoster infection earlier occurred. This tendency may be explained by the association between viral infection and development of SS [7]. 


\section{Disclosure Statement}

The authors have no conflicts of interest to declare.

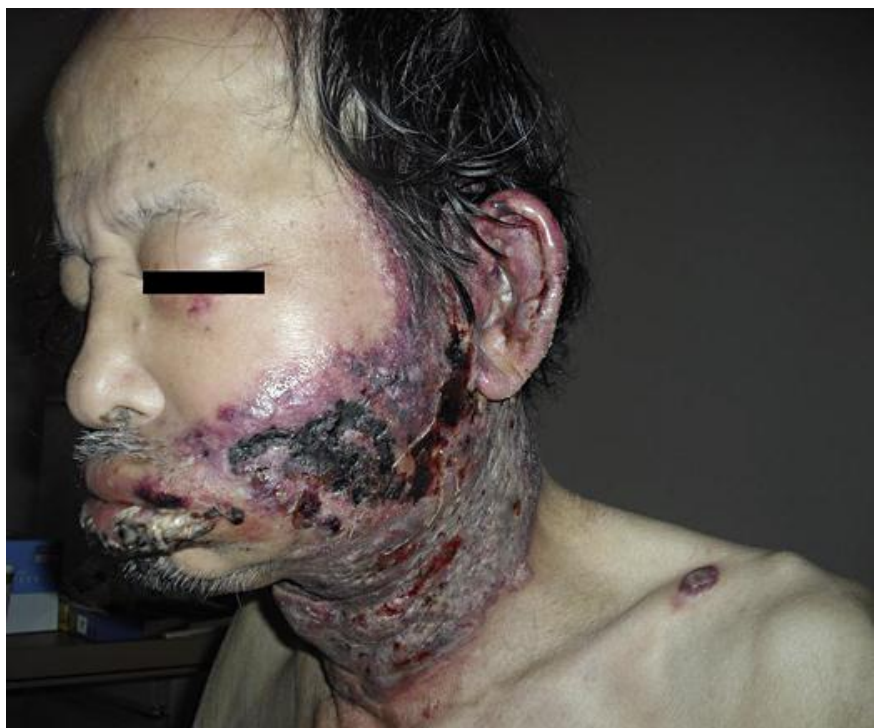

Fig. 1. Initial blisters developed abruptly, involving the left side of the oral mucosa and cheek. The lesions coalesced, forming a single large plaque covered with loose blisters on the cheek and neck.

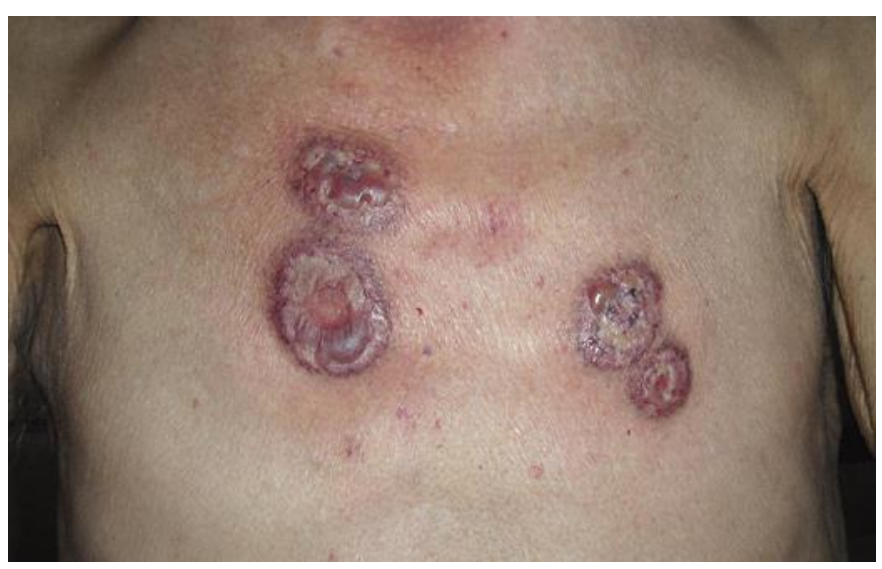

Fig. 2. Bullae on the trunk were isolated and circumscribed by small blisters. 


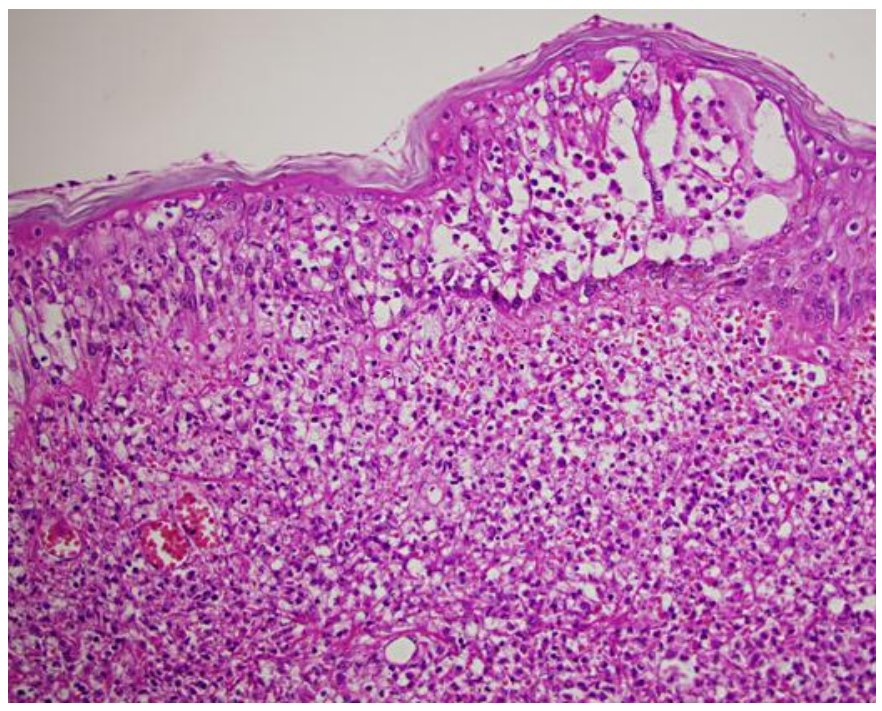

Fig. 3. Haematoxylin-eosin stain showing focal epidermal vesicles with neutrophilic microabscesses and interstitial neutrophilic dermal infiltrates with leukocytoclasis (original magnification $\times 200$ ).

\section{References}

1 Cohen PR, Kurzrock R: Sweet's syndrome revisited: a review of disease concepts. Int J Dermatol 2003;42:761-778.

-2 Voelter-Mahlknecht S, Bauer J, Metzler G, Fierlbeck G, Rassner G: Bullous variant of Sweet's syndrome. Int J Dermatol 2005;44:946-947.

3 Neoh CY, Tan AW, Ng SK: Sweet's syndrome: a spectrum of unusual clinical presentations and associations. Br J Dermatol 2007;156:480-485.

-4 Chiang CT, Chan HL, Kuo TT, Wang PN: Herpes zoster-like Sweet's syndrome in acute myelogenous leukemia. Int J Dermatol 1997;36:717-718.

-5 Török L, Kirschner A, Gurzó M, Krenács L: Bullous pyoderma gangrenosum as a manifestation of leukemia cutis. Eur J Dermatol 2000;10:463-465.

6 Guhl G, García-Díez A: Subcutaneous sweet syndrome. Dermatol Clin 2008;26:541-551.

$>7$ Choi JW, Chung KY: Sweet's syndrome with systemic lupus erythematosus and herpes zoster. Br J Dermatol 1999;140:1174-1175. 